\title{
Bursting Oscillation and Its Mechanism of a Generalized Duffing-Van der Pol System with Periodic Excitation
}

\author{
Youhua Qian (D), Danjin Zhang, and Bingwen Lin (iD) \\ College of Mathematics and Computer Science, Zhejiang Normal University, Jinhua, Zhejiang 321004, China \\ Correspondence should be addressed to Bingwen Lin; linbw921@zjnu.edu.cn
}

Received 26 February 2021; Revised 27 May 2021; Accepted 30 June 2021; Published 9 July 2021

Academic Editor: Pietro De Lellis

Copyright (c) 2021 Youhua Qian et al. This is an open access article distributed under the Creative Commons Attribution License, which permits unrestricted use, distribution, and reproduction in any medium, provided the original work is properly cited.

\begin{abstract}
The complex bursting oscillation and bifurcation mechanisms in coupling systems of different scales have been a hot spot domestically and overseas. In this paper, we analyze the bursting oscillation of a generalized Duffing-Van der Pol system with periodic excitation. Regarding this periodic excitation as a slow-varying parameter, the system can possess two time scales and the equilibrium curves and bifurcation analysis of the fast subsystem with slow-varying parameters are given. Through numerical simulations, we obtain four kinds of typical bursting oscillations, namely, symmetric fold/fold bursting, symmetric fold/supHopf bursting, symmetric subHopf/fold cycle bursting, and symmetric subHopf/subHopf bursting. It is found that these four kinds of bursting oscillations are symmetric. Combining the transformed phase portrait with bifurcation analysis, we can observe bursting oscillations obviously and further reveal bifurcation mechanisms of these four kinds of bursting oscillations.
\end{abstract}

\section{Introduction}

The multitime scale coupling dynamical systems involve mechanics, chemistry, biology, and so on and have a wide range of engineering backgrounds [1-9], for example, prototypical thermoacoustic system, the coupling between tower lines in the transmission tower system, reaction rates of different orders of magnitude existing in chemical reactions, allosteric effects in biological metabolism, and electrical activity of neuron bursting [10-15]. Obviously, there are a variety of factors that can lead to multitime scales coupling. However, the mathematical models of such systems and their oscillation characteristics still have certain similarities, which can be characterized by nonlinear dynamic systems $[16,17]$ with slow-varying parameters or slow variables. The multitime scales coupling system is usually characterized by the large amplitude oscillation and the small one, which can be regarded as the spiking state and the quiescent state of the system, respectively. A system exhibits bursting oscillations [1] if it has a periodic orbit $\gamma$ and the time series (in at least one fast phase space variable and in some coordinate scale) of $\gamma$ alternates between rapid oscillations and near steady-state behavior. Rinzel [18] proposed a slow-fast dynamics analysis method, which is a classical method to study the mechanism of bursting oscillation in two time scales. It reveals the mechanism of bursting oscillation in coupling systems in different time scales and points out that the essential reason for bursting oscillation is the mutual transition between the quiescent state and the spiking state. It is the core of the slow-fast dynamics analysis method to discuss the fast subsystem and the slow one separately. The fast subsystem has motion state of different modes, among which the spiking state corresponds to the large amplitude oscillation, while the quiescent state corresponds to the equilibrium state or the small amplitude oscillation. The slow subsystem can adjust the behavior of the fast subsystem to the whole system. When the slow variable passes through the motion mode of different regions in the fast subsystem periodically and slowly, the bursting oscillation often occurs. The introduction of the slow-fast dynamics analysis method [19] well explained the bifurcation connection between the spiking state and the quiescent state, so as the mechanism of bursting oscillation. Based on this method, various bursting oscillation modes [20-33] are obtained, such as fold/fold bursting and fold/ Hopf bursting. 
In nonlinear dynamical system, two time scales can lead to complex dynamical behavior of the system, so it is widely concerned by academic circles domestically and overseas. In recent decades, scholars have studied the dynamic behavior of two time scales from theoretical analysis and numerical simulation. Firstly, the approximate solution of the system is given by reducing the whole system to a single time scale. Next, the singular perturbation method $[34,35]$ is used to analyze the analytic solutions of multitime scales systems. However, neither of the two methods can deeply explore the complex dynamic characteristics generated by the interaction of different time scales. Until Rinzel proposed slow-fast analysis, the interaction between different time scales and the mechanism of bursting oscillations have been well revealed. For example, for the general slow-fast systems, Izhikevich [36] made a good summary of various bursting phenomena and bifurcation mechanisms in low-dimensional case. Saggio et al. [37] proposed a model that could display several types of bursting behaviors, consisting of two subsystems in different time scales, which provided a single framework to understand the coexistence of diverse bursting patterns in physical and biological systems or models. Zhang et al. [38] provided a method to explain the mechanism of bursting oscillation in a system with multiple periodic excitations. Moivre equation is used to convert two excitation terms into a function of a basic periodic excitation term, which is regarded as a slow-variable parameter. On the basis of those, bursting oscillation and its mechanism of system were analyzed. Lin et al. [39] established a mathematical model based on a three-element memristive circuit with slow-fast effect and discussed the mechanism of bursting oscillation using the slow-fast dynamics analysis method. Based on a three-dimensional nonautonomous system, Chen et al. [40] analyzed three modes of aperiodic polymetal oxide semiconductor under slow parameter excitation and discussed the mechanism of bursting oscillation. Zhang and $\mathrm{Bi}$ [41] investigated the normal form of a four-dimensional cubic order system with parametric excitation as a slow parameter, obtained several equilibrium branches and mechanisms of bursting oscillations for different amplitudes of the parametric excitation, and derived the mechanism of bursting oscillations.

Up to now, although some achievements have been made in the field of multitime scales research, most of the achievements are on low-dimensional systems, and the two time scales problems in high-dimensional systems are less involved. Because of the complexity of high-dimensional systems, such as the coexistence of multiple equilibrium states, the bursting behaviors of the system are particularly complex. Therefore, the multitime scales' problem of highdimensional system needs to be explored.

The remainder of this paper is organized as follows. In Section 2, the fold bifurcation set and Hopf bifurcation set are discussed by adjusting the value of parameters. In Section 3 , we analyze the bifurcation behaviors in two kinds of typical parameter sets. In Section 4, using the slow-fast dynamics analysis method, four kinds of typical bursting oscillations are emphatically analyzed. In Section 5, we make a conclusion.

\section{Mathematical Model and Bifurcation Set}

2.1. Mathematical Model. In this paper, we consider a kind of Duffing-Van der Pol system of slow-fast coupling:

$$
\left\{\begin{array}{l}
\dot{x}=y, \\
\dot{y}=u_{1} x+u_{2} y-x^{3}-x^{5}+a x^{2} y+b x^{4} y .
\end{array}\right.
$$

In order to study the bursting oscillation behavior of codimension one bifurcation system, we introduce a periodic excitation into system (1) and derive a new nonautonomous system:

$$
\left\{\begin{array}{l}
\dot{x}=y \\
\dot{y}=u_{1} x+u_{2} y-x^{3}-x^{5}+a x^{2} y+b x^{4} y+f \cos \omega t
\end{array}\right.
$$

where $f \cos \omega t$ denotes the periodic excitation ( $f$ is amplitude, and $\omega$ is frequency). Defining the new parameter, $F=f \cos \omega t$, system (2) becomes

$$
\left\{\begin{array}{l}
\dot{x}=y, \\
\dot{y}=u_{1} x+u_{2} y-x^{3}-x^{5}+a x^{2} y+b x^{4} y+F .
\end{array}\right.
$$

When there is an order of magnitude difference between the excitation frequency $\omega$ and the natural frequency $\Omega$ of the system, it has different time scales. Let $\omega \ll 1$, and it is clear that the system has a slow-fast coupling of two time scales. Regarding the periodic excitation $F$ as a generalized parameter of the system, system (3) can be called generalized autonomous system with respect to the slow variable $F$ accordingly. We regard the generalized autonomous system (3) as the fast subsystem corresponding to the fast variable $x$ and $y$, while treating $F=f \cos \omega t$ as the corresponding slow subsystem. $F$ plays a role in slow-varying adjustment for system (3), so the traditional slow-fast dynamics analysis method can be used to investigate the mechanism of different bursting oscillations in periodic excitation.

2.2. Bifurcation Set. We define the equilibrium point of the generalized autonomous system (3) as $E\left(x_{0}, 0\right)$, and its characteristic equation can be expressed as

$$
\lambda^{2}-\left(u_{2}+a x_{0}^{2}+b x_{0}^{4}\right) \lambda-\left(u_{1}-3 x_{0}^{2}-5 x_{0}^{4}\right)=0
$$

where $x_{0}$ satisfies

$$
u_{1} x_{0}-x_{0}^{3}-x_{0}^{5}+F=0 .
$$

According to Routh-Hurwitz criterion, the equilibrium point $E$ is stable under the following conditions:

$$
\left\{\begin{array}{l}
u_{2}+a x_{0}^{2}+b x_{0}^{4}<0 \\
u_{1}-3 x_{0}^{2}-5 x_{0}^{4}<0
\end{array}\right.
$$

By adjusting the value of parameters, the instability of the equilibrium point $E$ will lead to bifurcation of different forms; thus, we can obtain the possible fold bifurcation set and Hopf bifurcation set. 
Combining the characteristic equations (4) and (5), the condition for fold bifurcation to occur at $E$ can be obtained as follows:

$$
\left\{\begin{array}{l}
u_{1}-3 x_{0}^{2}-5 x_{0}^{4}=0 \\
u_{1} x_{0}-x_{0}^{3}-x_{0}^{5}+F=0
\end{array}\right.
$$

Fold bifurcation can cause the jumping phenomenon between different equilibrium points.

According to Hopf bifurcation theorem, we can obtain the conditions that Hopf bifurcation may occur in the system. Letting $\lambda= \pm i \omega_{0}$, substituting into characteristic equation (4), and eliminating $\omega_{0}$, then we can get the bifurcation conditions of Hopf bifurcation generated by $E$ :

$$
\left\{\begin{array}{l}
u_{2}+a x_{0}^{2}+b x_{0}^{4}=0, \\
u_{1} x_{0}-x_{0}^{3}-x_{0}^{5}+F=0, \\
u_{1}-3 x_{0}^{2}-5 x_{0}^{4}<0
\end{array}\right.
$$

Hopf bifurcation can cause the periodic bursting oscillation. So, the first condition of Hopf bifurcation theorem is satisfied.

Taking partial derivatives in both sides of the characteristic equation (4) with respect to $F$, we have

$$
\frac{\mathrm{d} \lambda}{\mathrm{d} F}=\frac{\left(\mathrm{d} x_{0} / \mathrm{d} F\right)\left(2 a x_{0} \lambda+4 b x_{0}^{3} \lambda-6 x_{0}-20 x_{0}^{3}\right)}{2 \lambda-\left(u_{2}+a x_{0}^{2}+b x_{0}^{4}\right)} .
$$

Substituting

$$
\frac{\mathrm{d} x_{0}}{\mathrm{~d} F}=\frac{1}{5 x_{0}^{4}+3 x_{0}^{2}-u_{1}},
$$

into equation (9) and letting $\lambda=i \omega_{0}$, we get

$$
\frac{\mathrm{d} \lambda}{\mathrm{d} F}=\frac{\left(2 a x_{0}+4 b x_{0}^{3}\right) i \omega_{0}-6 x_{0}-20 x_{0}^{3}}{\left(5 x_{0}^{4}+3 x_{0}^{2}-u_{1}\right)\left[2 i \omega_{0}-\left(u_{2}+a x_{0}^{2}+b x_{0}^{4}\right)\right]}
$$

Sorting out equation (11) and separating the real part of $(\mathrm{d} \lambda / \mathrm{d} F)$, we have

$$
\operatorname{Re}\left(\frac{\mathrm{d} \lambda}{\mathrm{d} F}\right)=\frac{\left(4 a x_{0}+8 b x_{0}^{3}\right) \omega_{0}^{2}+\left(6 x_{0}+20 x_{0}^{3}\right)\left(u_{2}+a x_{0}^{2}+b x_{0}^{4}\right)}{\left(5 x_{0}^{4}+3 x_{0}^{2}-u_{1}\right)\left[4 \omega_{0}^{2}+\left(u_{2}+a x_{0}^{2}+b x_{0}^{4}\right)^{2}\right]}
$$

Fixing the parameters, it can derive

$$
\operatorname{Re}\left(\frac{\mathrm{d} \lambda}{\mathrm{d} F}\right) \neq 0
$$

Therefore, the second condition of Hopf bifurcation theorem is also satisfied. So, it is proved that Hopf bifurcation will occur in the system, and the equilibrium point may be unstable due to Hopf bifurcation.

In order to judge the stability and bifurcation direction of the Hopf bifurcation, the first Lyapunov coefficient method is introduced. System (3) can be rewritten as $\dot{x}=A x+F(x), x \in R^{2}$, where $A$ is Jacobian matrix and can be represented as

$$
A=\left(\begin{array}{cc}
0 & 1 \\
u_{1}-3 x_{0}^{2}-5 x_{0}^{4} & u_{2}+a x_{0}^{2}+b x_{0}^{4}
\end{array}\right),
$$

$A q=i \omega_{0} q, A^{T} p=-i \omega_{0} p$, and $\langle p, q\rangle=\sum_{i=1}^{2} \overline{p_{i}} q_{i}=1$, where $A^{T}$ is the transposed matrix of $A$ and $\langle\cdot, \cdot\rangle$ is the standard scalar product in $R^{2} \cdot F(x)=O\left(\|x\|^{2}\right)$ is a smooth function. In the vicinity of $x=0$, its Taylor expansion is

$$
F(x)=\frac{1}{2} B(x, x)+\frac{1}{6} C(x, x, x)+O\left(\|x\|^{4}\right),
$$

where $B(x, x)$ and $C(x, x, x)$ are multilinear functions. In coordinates, we have

$$
\begin{gathered}
B_{i}(x, y)=\left.\sum_{j, k=1}^{2} \frac{\partial^{2} F_{i}(\xi)}{\partial \xi_{j} \partial \xi_{k}}\right|_{\xi=0} x_{j} y_{k}, \quad i=1,2, \\
C_{i}(x, y, z)=\left.\sum_{j, k, l=1}^{2} \frac{\partial^{3} F_{i}(\xi)}{\partial \xi_{j} \partial \xi_{k} \partial \xi_{l}}\right|_{\xi=0} x_{j} y_{k} z_{l}, \quad i=1,2 .
\end{gathered}
$$

So, the first Lyapunov coefficient

$$
l_{1}=\frac{1}{2 \omega_{0}} \operatorname{Re} G=-\frac{a}{2 \omega_{0}},
$$

where $\quad G=\langle p, C(q, q, \bar{q})\rangle-\quad 2\left\langle p, B\left(q, A^{-1} B(q, \bar{q})\right)\right\rangle+$ $\left\langle p, B\left(\bar{q},\left(2 i \omega_{0} E-A\right)^{-1} B(q, q)\right)\right\rangle$.

When the first Lyapunov coefficient $l_{1}<0$, a stable supercritical Hopf bifurcation occurs. When $l_{1}>0$, an unstable subcritical Hopf bifurcation occurs. When $l_{1}=0$, codimension-2 degenerate Hopf bifurcation occurs. So, when $a>0$, a stable supercritical Hopf bifurcation occurs. When $a<0$, an unstable subcritical Hopf bifurcation occurs.

For the above two kinds of different bifurcation sets (7) and (8), the generalized autonomous system will produce bifurcation in different forms. When the bursting oscillation phenomenon occurs, it corresponds to the transition point between the quiescent state and the spiking state.

\section{Bifurcation Behavior}

To study the dynamic behavior under the parameters $u_{1}$ and $F$, we need to fix the parameters $u_{2}, a$, and $b$. Through analysis, we consider two typical cases, whose stability of equilibrium points is different:

(I) $u_{2}=-0.1, a=0.1$, and $b=0.1$

(II) $u_{2}=2, a=-2$, and $b=-2$

Different situations may give birth to different number of equilibrium points and the related bifurcation types in a generalized autonomous system.

3.1. The Bifurcation Behavior under Case I. Let $u_{1}$ and $F$ be bifurcation parameters. Figure 1(a) shows the two-parameter bifurcation sets on the $F-u_{1}$ plane. It divides the parameter plane $\left(F, u_{1}\right)$ into seven regions, and each region corresponds to solutions of different forms of the system. We consider the bifurcation behaviors when $u_{1}=-0.4,0.4$, 0.8 , and 4.0 . 


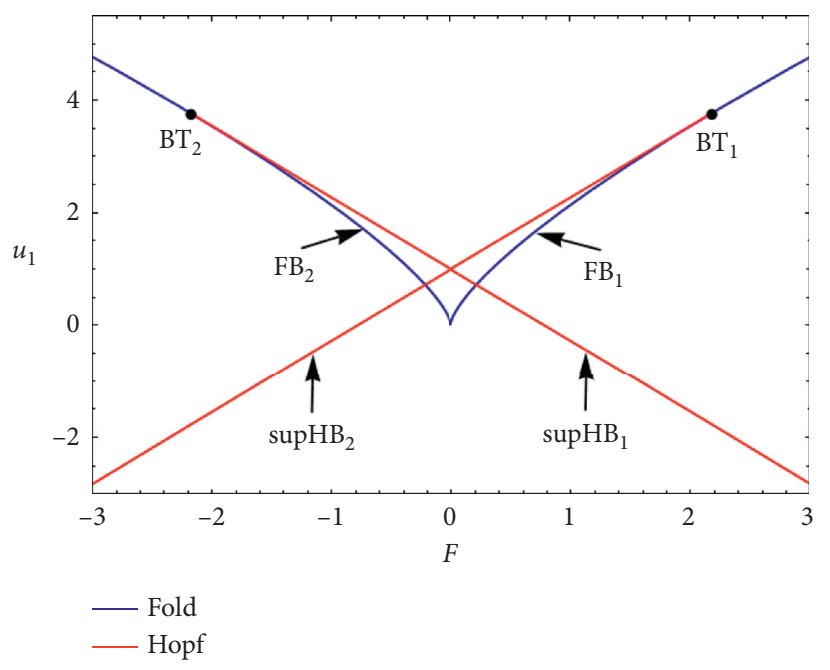

(a)

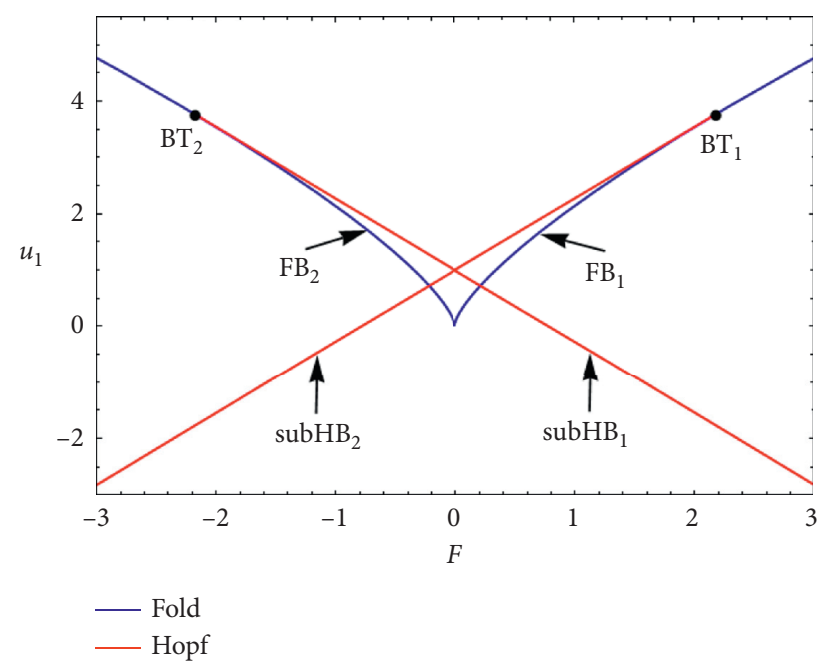

(b)

Figure 1: Two-parameter bifurcation sets in $\left(F, u_{1}\right)$. (a) Case I. (b) Case II. BT 1 and $\mathrm{BT}_{2}$ represent the two Bogdanov-Takens bifurcation points, the blue solid line represents the fold bifurcation curve, and the red solid line represents the Hopf bifurcation curves.

Figure 2(a) shows equilibrium curve with respect to the slow-varying parameter $F$ for $u_{1}=-0.4$, wherein two Hopf bifurcation points $\left(\operatorname{supHB}_{1}\right.$ and $\left.\operatorname{supHB}_{2}\right)$ are located at $(1.1006,0.7862)$ and $(-1.1006,-0.7862)$. In this figure, solid and imaginary lines represent stable and unstable equilibrium points, respectively. The green solid circles represent stable limit cycles, and the blue hollow circles represent unstable limit cycles. When $F=1.2$, the slow-varying parameter $F$ varies from -1.2 to 1.2 . Thus, $F$ only traverses the two Hopf bifurcation points $(F= \pm 1.1006)$. Periodic oscillation can occur this time, but no bursting oscillation occurs.

Figure 2(b) shows equilibrium curve with respect to the slow-varying parameter $F$ for $u_{1}=0.4$, wherein two Hopf bifurcation points $\left(\operatorname{supHB}_{1}\right.$ and $\left.\operatorname{supHB}_{2}\right)$ are located at $(0.4717,0.7862)$ and $(-0.4717,-0.7862)$, and twofold bifurcation points $\left(\mathrm{FB}_{1}\right.$ and $\left.\mathrm{FB}_{2}\right)$ are located at $(0.0922,-0.3351)$ and $(-0.0922,0.3351)$. When $F=0.2$, the slow-varying parameter $F$ varies from -0.2 to 0.2 . Thus, Fonly traverses the twofold bifurcation points $(F= \pm 0.0922)$. Under this condition, jumping phenomenon may take place when the slow-varying parameter $F$ arrives at points $\mathrm{FB}_{1}$ and $\mathrm{FB}_{2}$. So symmetric fold/fold bursting occurs when $F=0.2$. When $F=0.5$, the slowvarying parameter $F$ varies from -0.5 to 0.5 . Thus, $F$ can traverse the twofold bifurcation points $(F= \pm 0.0922)$ and two Hopf bifurcation points $(F= \pm 0.4717)$. Thus, symmetric fold/supHopf bursting occurs when $F=0.5$.

Figure 2(c) shows equilibrium curve with respect to the slow-varying parameter $F$ for $u_{1}=0.8$, wherein two Hopf bifurcation points $\left(\operatorname{supHB}{ }_{1}\right.$ and $\left.\operatorname{supHB}_{2}\right)$ are located at $(0.1572,0.7862)$ and $(-0.1572,-0.7862)$, and twofold bifurcation points $\left(\mathrm{FB}_{1}\right.$ and $\left.\mathrm{FB}_{2}\right)$ are located at $(0.2504,-0.4472)$ and $(-0.2504,0.4472)$. When $F=0.2$, the slow-varying parameter $F$ varies from -0.2 to 0.2 . Thus, $F$ only traverses the two Hopf bifurcation points $(F= \pm 0.1572)$. Periodic oscillation can also occur this time, but no bursting oscillation occurs. When $F=0.3$, the slow-varying parameter $F$ varies from -0.3 to 0.3 . Thus, $F$ can traverse the twofold bifurcation points $(F= \pm 0.2504)$ and two Hopf bifurcation points $(F= \pm 0.1572)$. Thus, symmetric fold/supHopf bursting occurs when $F=0.3$.

Figure 2(d) shows equilibrium curve with respect to the slow-varying parameter $F$ for $u_{1}=4.0$, wherein twofold bifurcation points $\left(\mathrm{FB}_{1}\right.$ and $\left.\mathrm{FB}_{2}\right)$ are located at $(2.3604,-0.8021)$ and $(-2.3604,0.8021)$. Under this condition, no oscillation occurs.

3.2. The Bifurcation Behavior under Case II. The two-parameter bifurcation sets of case I are similar to that of case II. Figure 1(b) shows the two-parameter bifurcation sets on the $F-u_{1}$ plane. We consider the bifurcation behaviors when $u_{1}=-0.4,0.7,0.9$, and 4.0 .

Figure 3(a) shows equilibrium curve with respect to the slow-varying parameter $F$ for $u_{1}=-0.4$, wherein two Hopf bifurcation points $\left(\mathrm{subHB}_{1}\right.$ and $\left.\mathrm{subHB}_{2}\right)$ are located at $(1.1006,0.7862)$ and $(-1.1006,-0.7862)$. In this figure, solid and imaginary lines represent stable and unstable equilibrium points, respectively. The green solid circles represent stable limit cycles, and the blue hollow circles represent unstable limit cycles. When $F=1.5$, the slowvarying parameter $F$ varies from -1.5 to 1.5 . Thus, $F$ only traverses the two Hopf bifurcation points $(F= \pm 1.1006)$. Hence, symmetric subHopf/fold cycle bursting occurs when $F=1.5$.

Figure 3(b) shows equilibrium curve with respect to the slow-varying parameter $F$ for $u_{1}=0.7$, wherein two Hopf bifurcation points $\left(\mathrm{subHB}_{1}\right.$ and $\left.\mathrm{subHB}_{2}\right)$ are located at $(0.2358,0.7862)$ and $(-0.2358,-0.7862)$, and twofold bifurcation points $\left(\mathrm{FB}_{1}\right.$ and $\left.\mathrm{FB}_{2}\right)$ are located at $(0.2069,-0.4238)$ and $(-0.2069,0.4238)$. When $F=1.0$, the slow-varying parameter $F$ varies from -1.0 to 1.0. Thus, $F$ traverses the twofold bifurcation points $(F= \pm 0.2069)$ and 


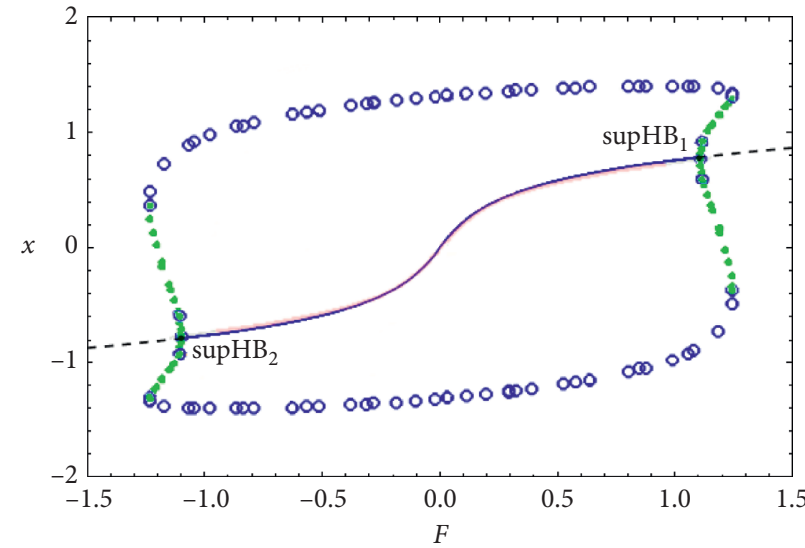

(a)

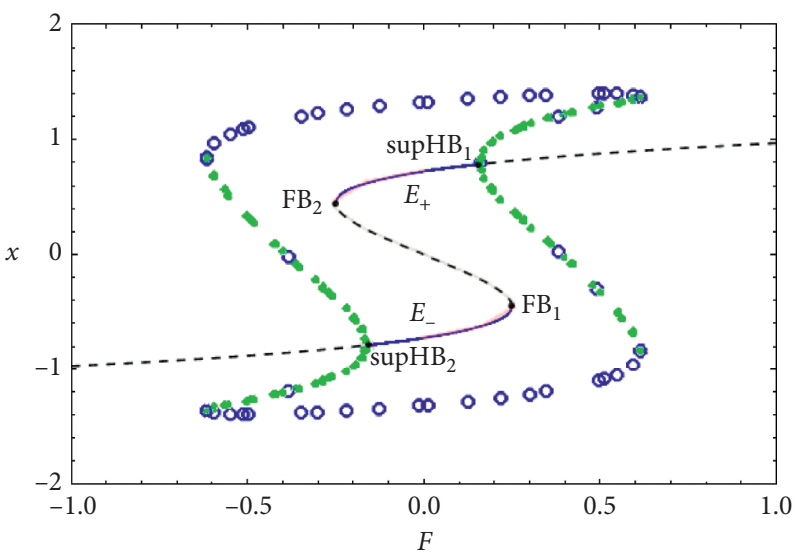

(c)

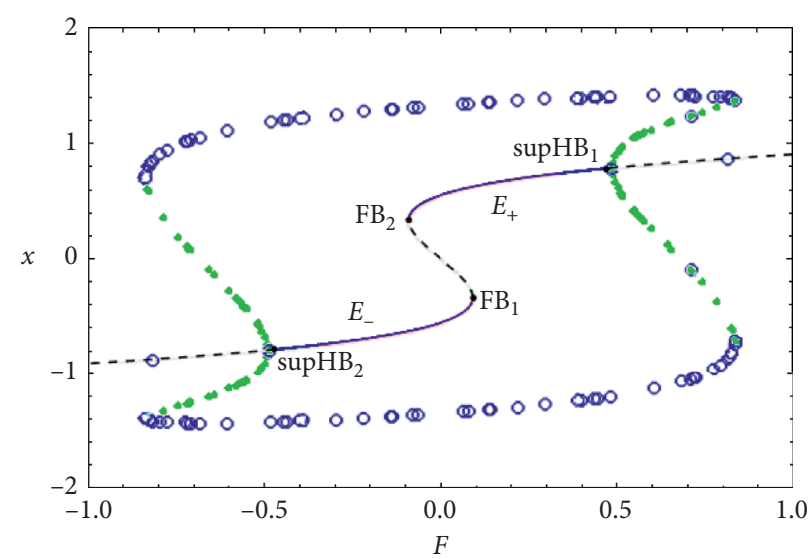

(b)

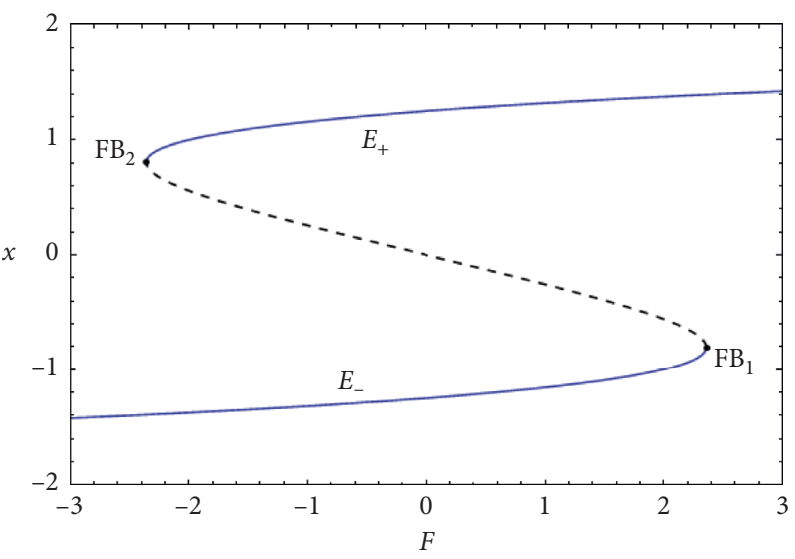

(d)

FIGURE 2: Equilibrium curves for case I. (a) $u_{1}=-0.4$. (b) $u_{1}=0.4$. (c) $u_{1}=0.8$. (d) $u_{1}=4.0$.

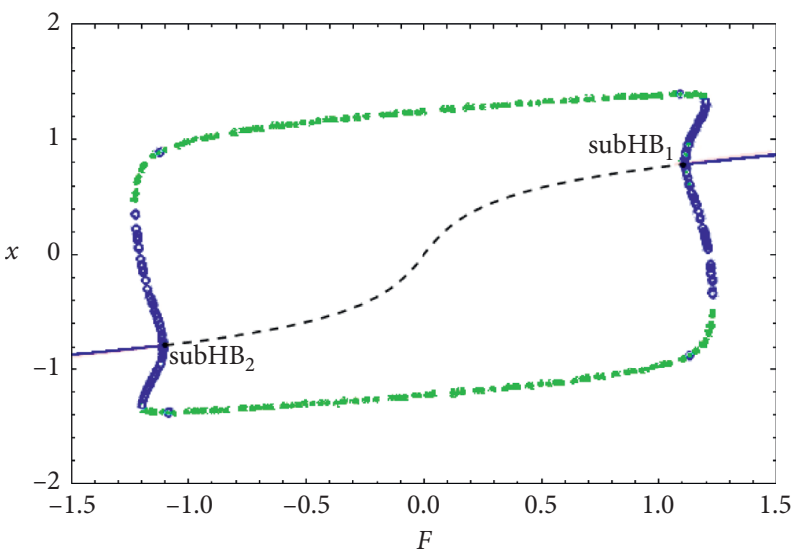

(a)

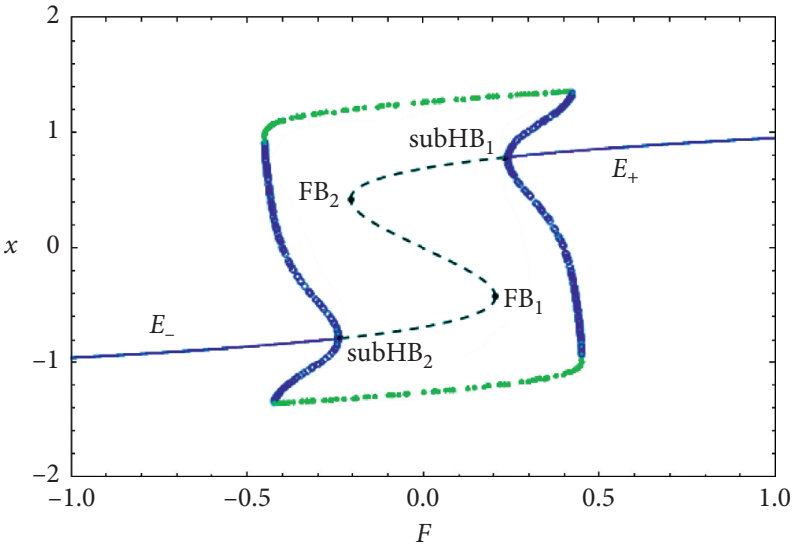

(b)

Figure 3: Continued. 


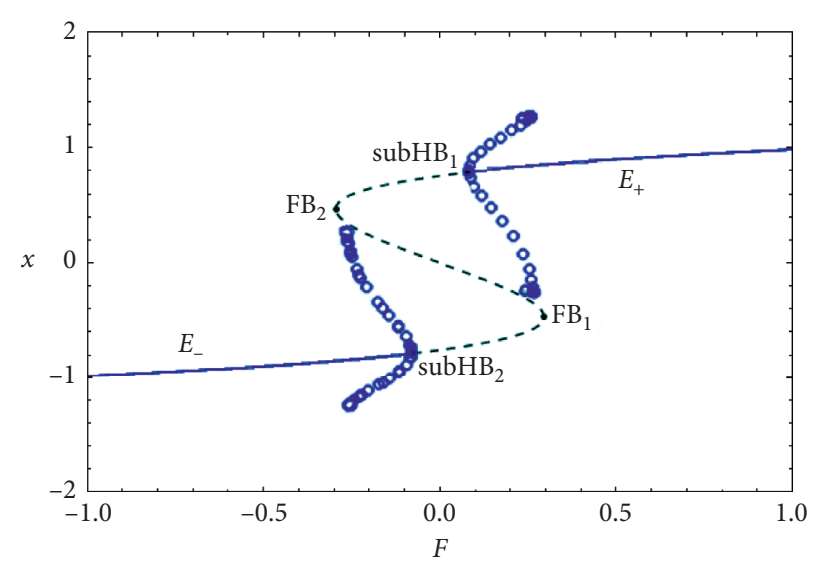

(c)

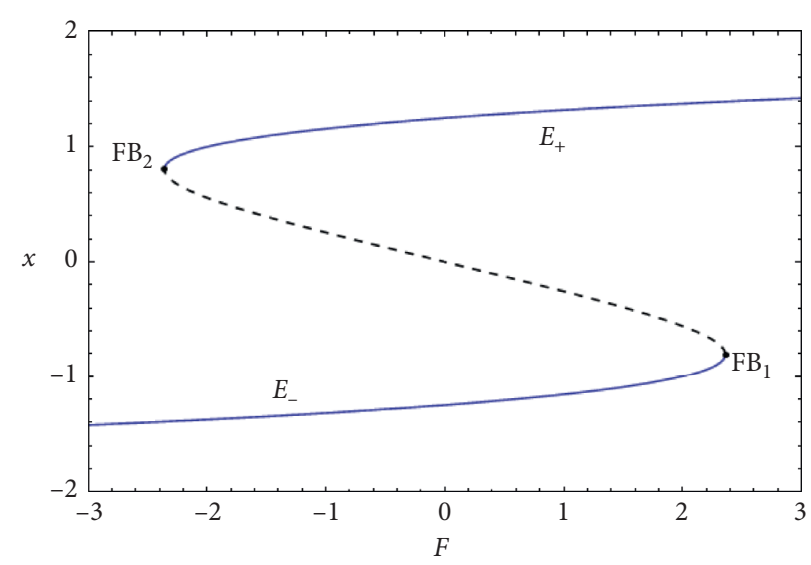

(d)

FIGURE 3: Equilibrium curves for case II. (a) $u_{1}=-0.4$. (b) $u_{1}=0.7$. (c) $u_{1}=0.9$. (d) $u_{1}=4.0$.

two Hopf bifurcation points $(F= \pm 0.2358)$. So symmetric subHopf/fold cycle bursting occurs when $F=1.0$.

Figure 3(c) shows equilibrium curve with respect to the slow-varying parameter $F$ for $u_{1}=0.9$, wherein two Hopf bifurcation points $\left(\mathrm{subHB}_{1}\right.$ and $\left.\mathrm{subHB}_{2}\right)$ are located at $(0.0786,0.7862)$ and $(-0.0786,-0.7862)$, and twofold bifurcation points $\left(\mathrm{FB}_{1}\right.$ and $\left.\mathrm{FB}_{2}\right)$ are located at $(0.2962,-0.4686)$ and $(-0.2962,0.4686)$. When $F=1.0$, the slow-varying parameter $F$ varies from -1.0 to 1.0 . Thus, $F$ can traverse the twofold bifurcation points $(F= \pm 0.2962)$ and two Hopf bifurcation points $(F= \pm 0.0786)$. Thus, symmetric subHopf/subHopf bursting occurs when $F=1.0$.

Figure 3(d) shows equilibrium curve with respect to the slow-varying parameter $F$ for $u_{1}=4.0$, wherein twofold bifurcation points $\left(\mathrm{FB}_{1}\right.$ and $\left.\mathrm{FB}_{2}\right)$ are located at $(2.3604,-0.8021)$ and $(-2.3604,0.8021)$. When $F=3.0$, the slow-varying parameter $F$ varies from -3.0 to 3.0. Thus, $F$ only traverses the twofold bifurcation points $(F= \pm 2.3604)$. Under this condition, jumping phenomenon may take place when the slow-varying parameter $F$ arrives at points $\mathrm{FB}_{1}$ and $\mathrm{FB}_{2}$. So symmetric fold/fold bursting occurs when $F=3.0$.

Compared with Figure 2, it is not difficult to find that, although the number of fold bifurcation points and Hopf bifurcation points are the same, their stabilities are different.

To present intuitively the bursting types in the system, Table 1 summarizes all results above.

\section{Bursting Oscillation Mechanisms}

Letting excitation frequency $\omega=0.01$, there are two time scales in the system, so as to study its dynamic behavior. In the sequel, we will discuss the evolution process of the system dynamics under cases I and II.

Since the above equilibrium points and their bifurcation analyses are all obtained based on parameter $F$, that is to say, the positions of equilibrium points and their bifurcation behavior under different $F$ values are given, the traditional phase portrait shows the relationship between different states changes. In order to reveal the relationship
TABLE 1: Bursting types of the system.

\begin{tabular}{cccc}
\hline Case I & $u_{1}=0.4$ & $\begin{array}{l}f=0.2 \\
f=0.5\end{array}$ & $\begin{array}{c}\text { Symmetric fold/fold bursting } \\
\text { Symmetric fold/supHopf bursting }\end{array}$ \\
\hline & $u_{1}=0.7$ & $f=1.0$ & $\begin{array}{c}\text { Symmetric subHopf/fold cycle } \\
\text { bursting }\end{array}$ \\
Case II & $u_{1}=0.9 \quad f=1.0$ & $\begin{array}{c}\text { Symmetric subHopf/subHopf } \\
\text { bursting }\end{array}$ \\
\hline
\end{tabular}

between state variables and $F$, the concept of the transformed phase portrait is introduced here. That is, the system trajectory is projected onto the $(F, x)=(f \cos \omega t, x(t))$ plane to describe the relationship between state variables and $F$. With the help of the transformed phase portrait and the bifurcation analysis of the fast subsystem, the effects of different equilibrium states and their bifurcations on bursting oscillation can be obtained, thus the mechanism of bursting oscillation can be revealed.

\subsection{Case $I: u_{2}=-0.1$ and $a=b=0.1$}

4.1.1. Symmetric Fold/Fold Bursting. By analyses above, we know that the system has two kinds of typical bifurcations under situation I, namely, fold bifurcation and Hopf bifurcation. In addition, as $F$ changes, the number of equilibrium points changes accordingly. Next, we set $u_{1}=0.4$ and $f=0.2$ to study the bursting oscillation and its mechanism. The phase portrait and the corresponding time history of variable $x$ are demonstrated in Figures 4(a) and 4(b). Here, the motion of the trajectory can be divided into four parts, including two quiescent states and two spiking states.

To further reveal the bifurcation mechanism of the bursting oscillation, Figure 4(d) shows the overlap portrait of equilibrium curve and transformed phase portrait on the $(F, x)$ plane. Assuming that the trajectory starts from point $\mathrm{A}$, it is attracted by the stable equilibrium point $E_{+}$, resulting in the fact that the trajectory moves to the left strictly along the stable equilibrium curve $E_{+}$with the decrease of $F$. At 


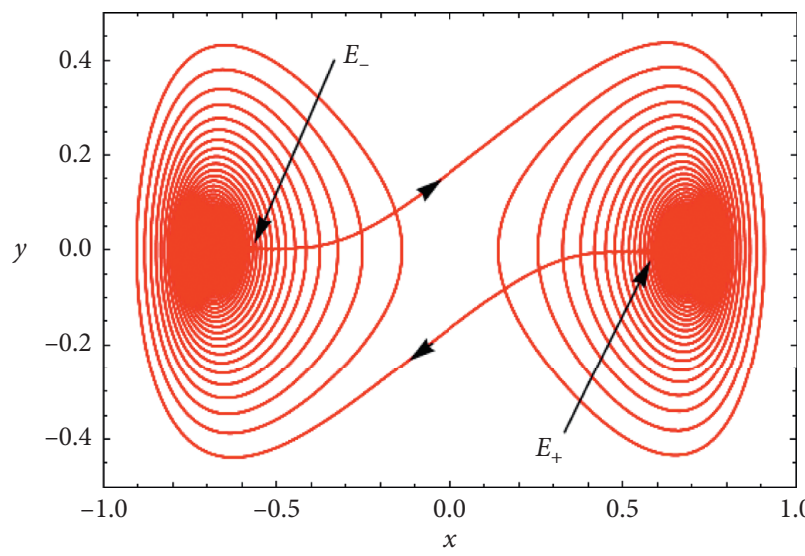

(a)

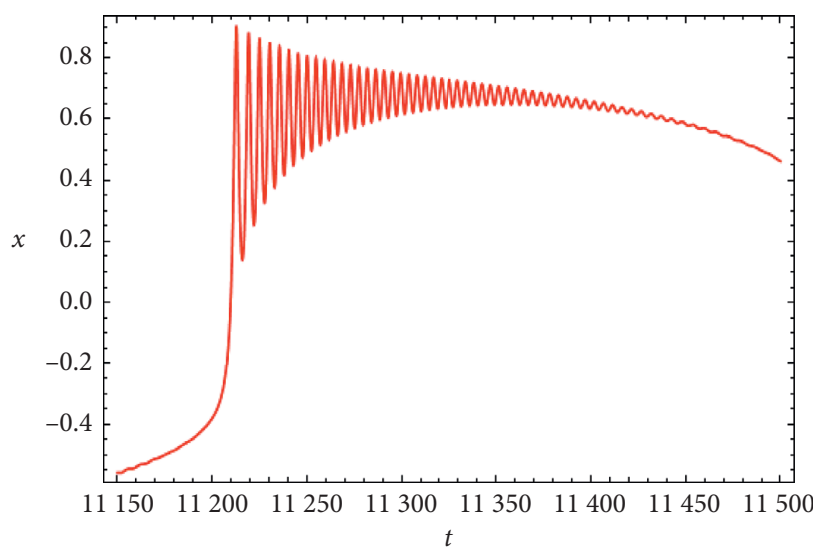

(c)

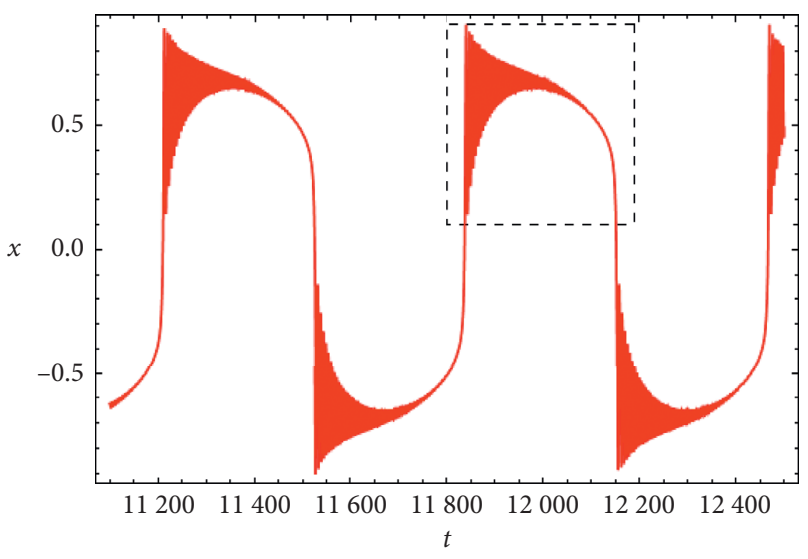

(b)

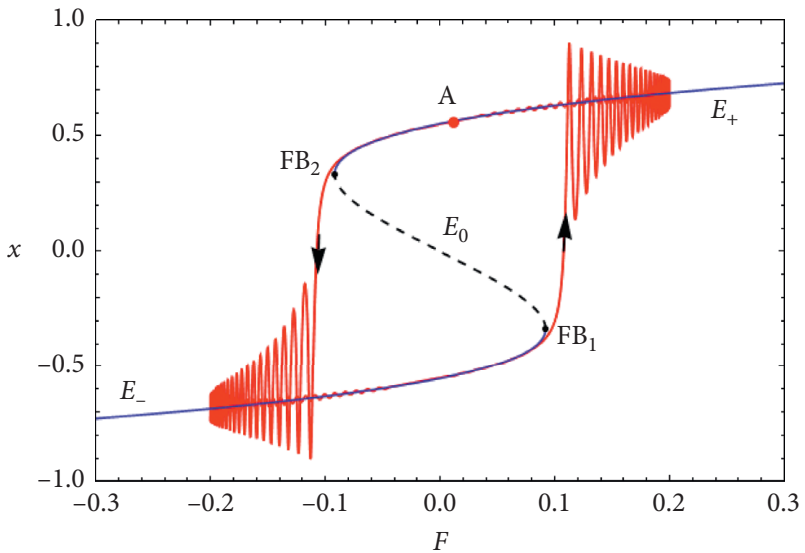

(d)

FIGURE 4: Symmetric fold/fold bursting oscillators for $u_{1}=0.4$ and $f=0.2$. (a) Phase portrait. (b) Time history of $x$. (c) Locally enlarged part of the (b). (d) The overlap of equilibrium curve and transformed phase portrait.

this time, the system remains in the quiescent state until arriving at the fold bifurcation point $\mathrm{FB}_{2}(F=-0.0922)$. Due to the fast subsystem's fold bifurcation, the jumping phenomenon occurs, and the equilibrium point $E_{+}$disappears. The trajectory jumps to $E_{-}$due to the attraction of the stable equilibrium point $E_{-}$and enters the spiking state SP from the quiescent state QS. As $F$ decreases continually, the trajectory gradually converges to the equilibrium point $E_{-}$ and enters the quiescent state QS. When the variable $F$ continues to decrease and meets its minimum -0.2 , the trajectory begins to move to the right under the action of external excitation.

Similarly, due to the symmetry of the phase space, the oscillation mechanism of the trajectory moving to the right is the same as above. Until returning to the initial point $\mathrm{A}$, a period of bursting oscillation is completed.

For the whole process of bursting oscillation period, the trajectory has experienced the conversion between two quiescent states and two spiking states, respectively, and the conversion between quiescent states and spiking states is caused by fold bifurcation. So, the dynamical behavior can be called symmetric fold/fold bursting.

It must be pointed out that the transformed phase portrait in Figure $4(\mathrm{~d})$ is introduced to explain the mechanism of bursting oscillation, while Figures 4(a) and 4(b) truly reflect the system's trajectory phase portrait, time history, and the phenomenon of bursting oscillation.

4.1.2. Symmetric Fold/supHopf Bursting. We set $u_{1}=0.4$ and $f=0.5$ to study the bursting oscillation and its mechanism. The phase portrait and the corresponding time history of variable $x$ are demonstrated in Figures 5(a) and 5(b). Here, the motion of the trajectory can be divided into four parts, including two quiescent states and two spiking states.

To further reveal the bifurcation mechanism of the bursting oscillation, Figure 5(d) shows the overlap portrait of equilibrium curve and transformed phase portrait on the $(F, x)$ plane. Assuming that the trajectory starts from point $\mathrm{A}$, it is attracted by the stable equilibrium point $E_{+}$, resulting in the fact that the trajectory moves to the left strictly along the stable equilibrium curve $E_{+}$with the decrease of $F$. At this time, the system remains in the quiescent state QS until arriving at the fold bifurcation point $\mathrm{FB}_{2}(F=-0.0922)$. Due to the fast subsystem's fold bifurcation, the jumping phenomenon occurs, and the equilibrium point $E_{+}$disappears. The trajectory jumps to $E_{-}$due to the attraction of the stable 


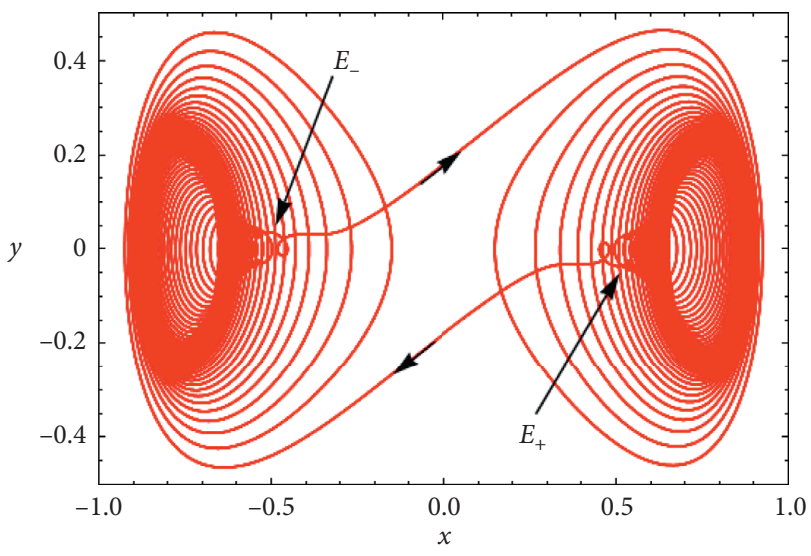

(a)

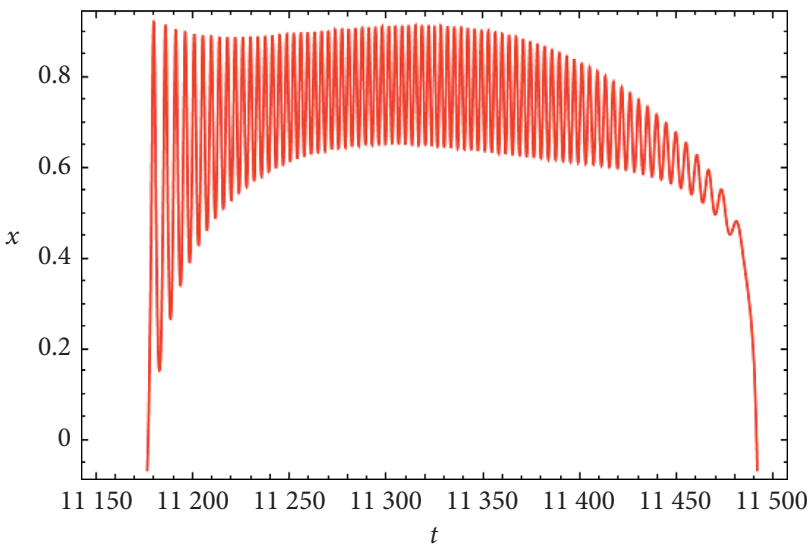

(c)

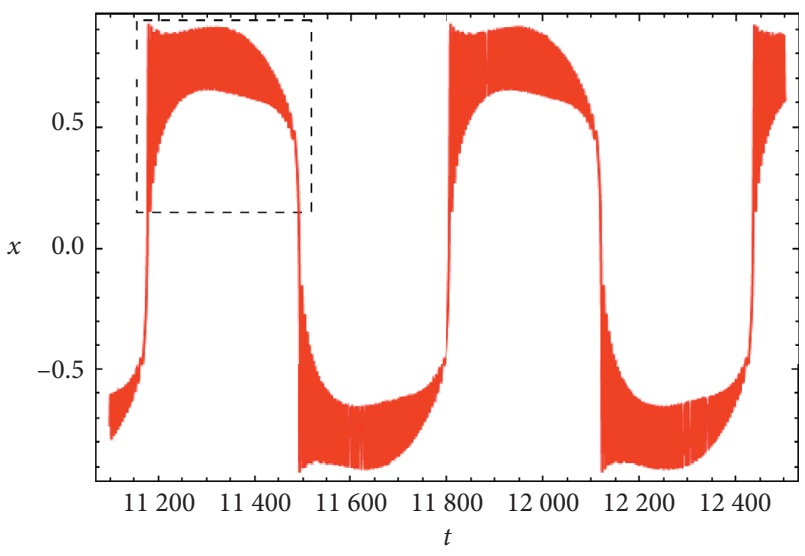

(b)

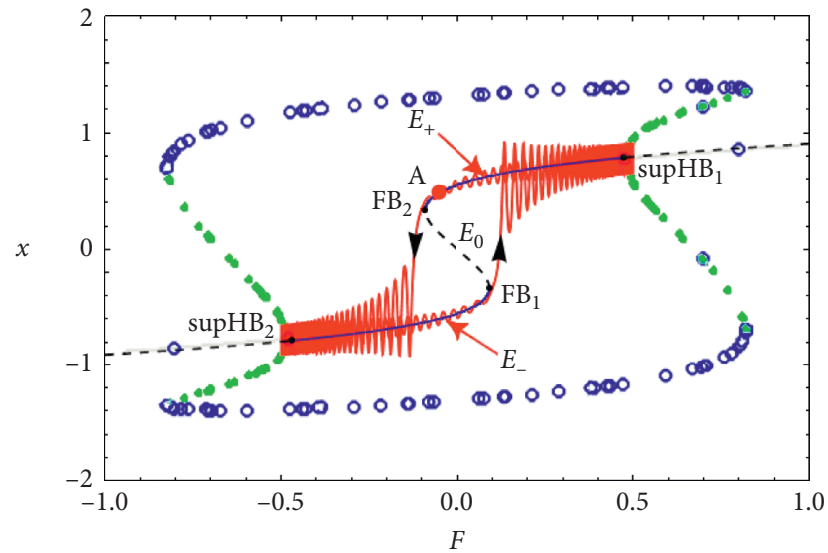

(d)

Figure 5: Symmetric fold/supHopf bursting oscillators for $u_{1}=0.4$ and $f=0.5$. (a) Phase portrait. (b) Time history of $x$. (c) Locally enlarged part of the (b). (d) The overlap of equilibrium curve and transformed phase portrait.

equilibrium point $E_{-}$and enters the spiking state SP from the quiescent state QS. As $F$ decreases continually to the supercritical Hopf bifurcation point $\operatorname{supHB}_{2}(F=-0.4717)$, Hopf bifurcation occurs and a stable limit cycle is observed. So, the trajectory moves around the stable limit cycle. When the variable $F$ continues to decrease and meets its minimum -0.5 , the trajectory begins to move to the right under the action of external excitation. Then, when the trajectory reaches supHB $\mathrm{H}_{2}$ again, the stable limit cycle disappears and the trajectory is attracted by the stable equilibrium point $E_{-}$. The trajectory gradually converges to the equilibrium point $E_{-}$and enters the quiescent state QS from the spiking state SP.

Similarly, due to the symmetry of the phase space, the oscillation mechanism of the trajectory moving to the right is the same as above. Until returning to the initial point $\mathrm{A}$, a period of bursting oscillation is completed.

For the whole process of bursting oscillation period, the trajectory has experienced the conversion between two quiescent states and two spiking states, respectively, and the conversion between quiescent states and spiking states is caused by fold bifurcation and supercritical Hopf bifurcation. So, the dynamical behavior can be called symmetric fold/supHopf bursting.

\subsection{Case II: $u_{2}=2$ and $a=b=-2$}

4.2.1. Symmetric subHopf/Fold Cycle Bursting. We set $u_{1}=$ 0.7 and $f=1.0$ to study the bursting oscillation and its mechanism. The phase portrait and the corresponding time history of variable $x$ are demonstrated in Figures 6(a) and 6(b).

Compared to the case previously, it is not difficult to find that although the motion process of the trajectory can still be divided into four parts, each spiking state contains two parts, namely, large amplitude oscillation $\mathrm{SP}_{1}$ and small amplitude oscillation $\mathrm{SP}_{2}$. In addition, bursting oscillation in a period is also similar to Figure 5. But it is worth noting that, as shown in Figure 6(c), when the trajectory reaches the breaking point $S$ and enters the spiking states, it oscillates around the stable limit cycle CY of generalized autonomous systems (as shown in Figure 7). And it does not jump to the stable equilibrium branch $E_{-}$by the unstable equilibrium branch $E_{+}$, which causes large amplitude oscillation. It is only when five large amplitude oscillations are finished that the trajectory begins to tend to the equilibrium branch $E_{-}$, resulting in small amplitude oscillation $\mathrm{SP}_{2}$.

To clearly illustrate the change of limit cycle with the variable $F$, Table 2 shows the distribution interval of stable 


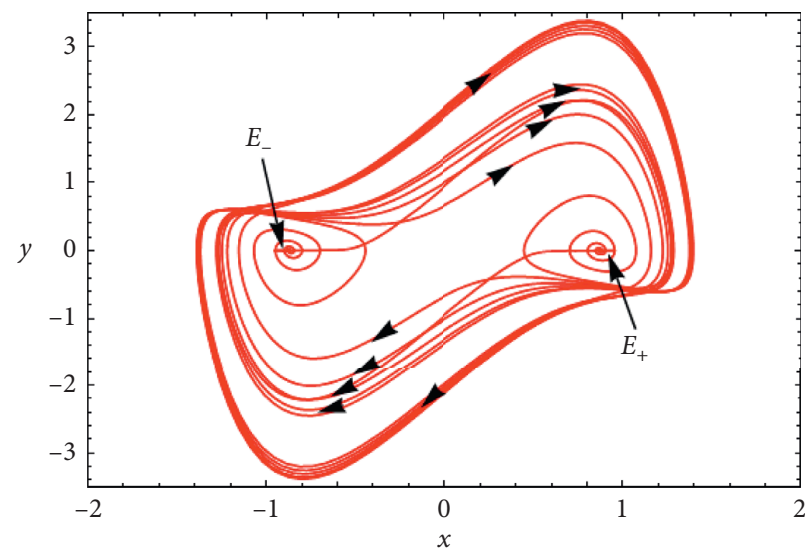

(a)

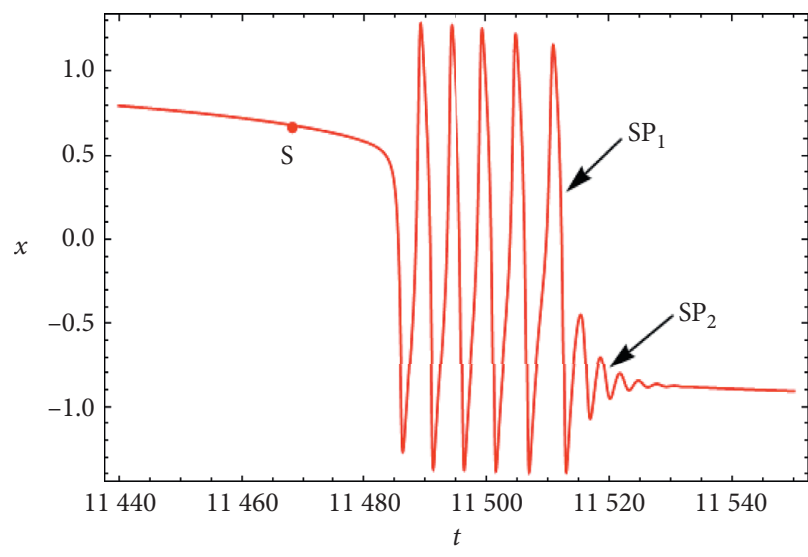

(c)

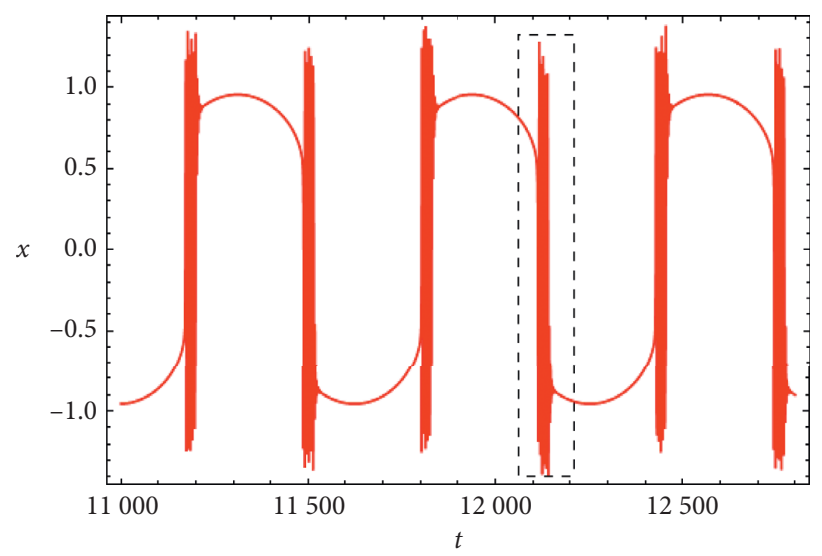

(b)

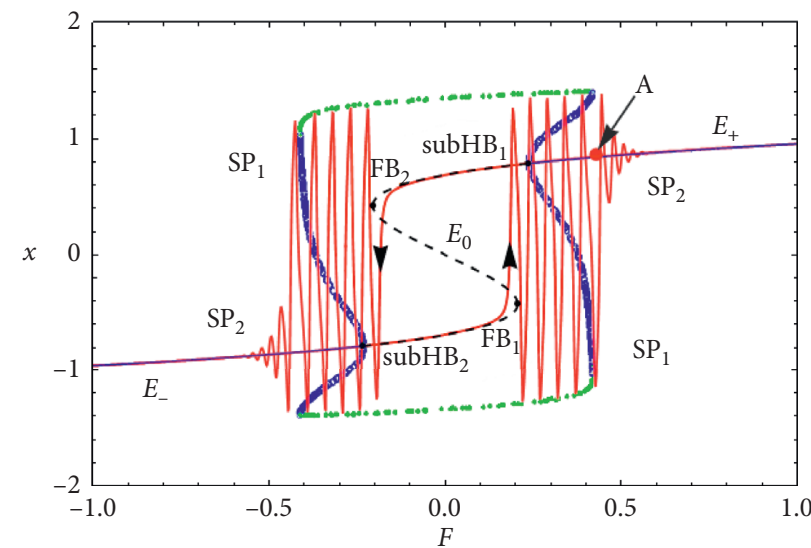

(d)

Figure 6: Symmetric subHopf/fold cycle bursting oscillators for $u_{1}=0.7$ and $f=1.0$. (a) Phase portrait. (b) Time history of $x$. (c) Locally enlarged part of the (b). (d) The overlap of equilibrium curve and transformed phase portrait.

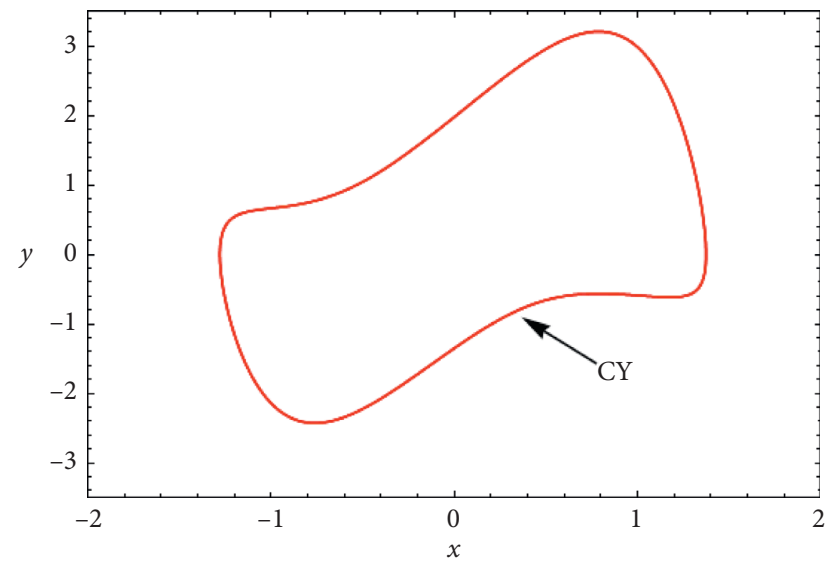

FIGURE 7: Stable limit cycle of generalized autonomous system for $u_{1}=0.7$ and $f=1.0$.

equilibrium points and limit cycle. Figure 6(d) shows the overlap portrait of equilibrium curve and transformed phase portrait on the $(F, x)$ plane.

As shown in Figure 6(d), assuming that the trajectory starts from point $\mathrm{A}$, it is attracted by the stable equilibrium
TABLE 2: Equilibrium points and limit cycle with variation of $F$ for $u_{1}=0.7$ in symmetric subHopf/fold cycle bursting oscillators.

\begin{tabular}{lccc}
\hline \multirow{2}{*}{ Stable equilibrium points } & \multicolumn{2}{c}{ Limit cycles } \\
& & Stable & Unstable \\
\hline$(-1.0,-0.3848)$ & $E_{-}$ & No & No \\
{$[-0.3848,-0.2358]$} & $E_{-}$ & Yes & Yes \\
{$[-0.2358,0.2358]$} & None & Yes & No \\
{$[0.2358,0.3609]$} & $E_{+}$ & Yes & Yes \\
$(0.3609,1.0)$ & $E_{+}$ & No & No \\
\hline
\end{tabular}

point $E_{+}$. So, the trajectory moves to the left strictly along the stable equilibrium curve $E_{+}$with the decrease of $F$. At this time, the system remains in the quiescent state QS. When the trajectory moves to the subcritical Hopf bifurcation point $\operatorname{subHB}_{1}(F=0.2358)$, the equilibrium point $E_{+}$loses its stability. But it still goes for a period of time along the unstable equilibrium point $E_{+}$due to the delay phenomenon. Until $F=-0.1320$, the jumping phenomenon occurs. We can observe that there is a stable limit cycle CY and a stable equilibrium point $E_{-}$at $F=-0.1320$. However, the trajectory is in the attraction domain of the limit cycle CY at this time. So, the trajectory is making large amplitude 


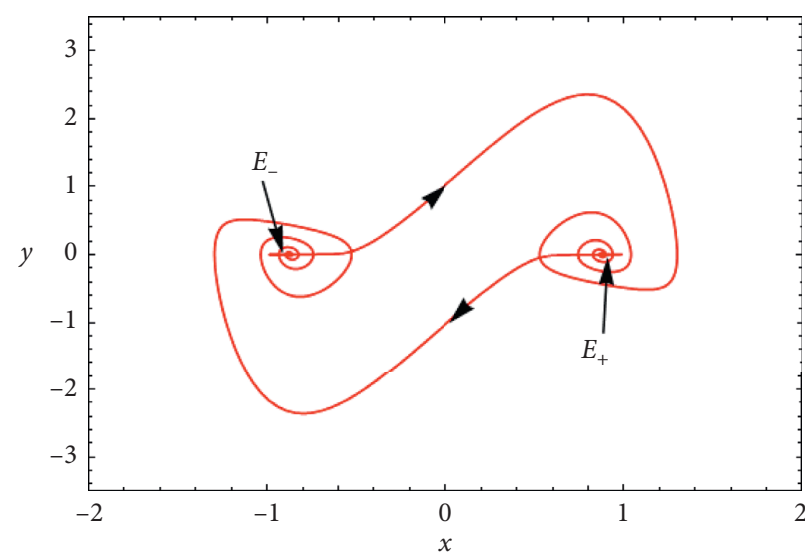

(a)

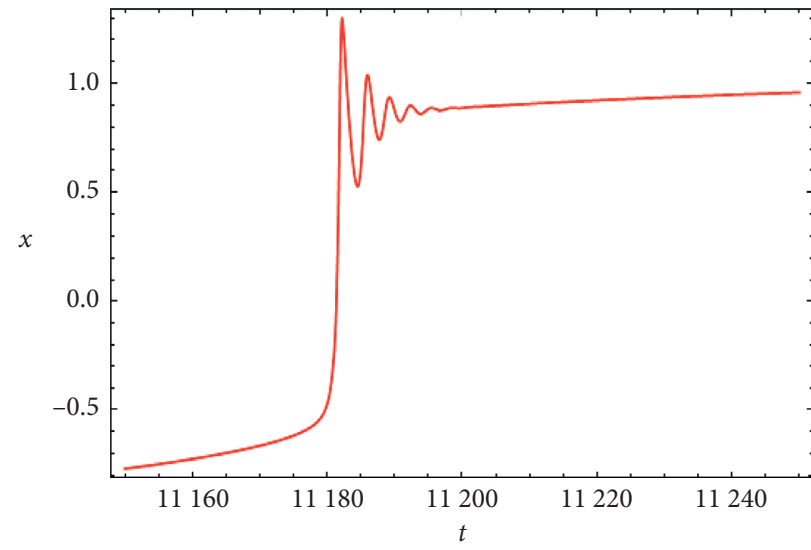

(c)

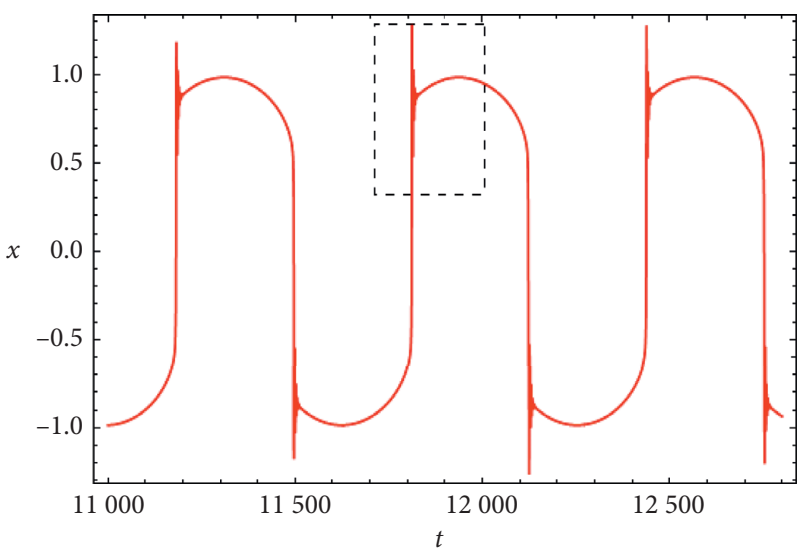

(b)

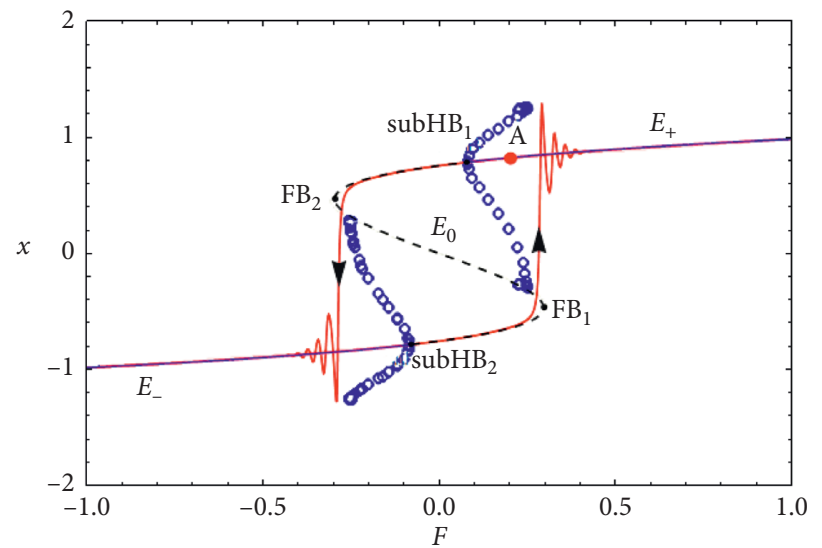

(d)

Figure 8: Symmetric subHopf/subHopf bursting oscillators for $u_{1}=0.9$ and $f=1.0$. (a) Phase portrait. (b) Time history of $x$. (c) Locally enlarged part of the (b). (d) The overlap of equilibrium curve and transformed phase portrait.

oscillation around the limit cycle CY, entering the spiking state $S P_{1}$ from the quiescent state QS. Until the stable limit cycle disappears, the trajectory begins to approach the stable equilibrium point $E_{-}$. The trajectory begins to make small amplitude oscillation around $E_{-}$, entering the spiking state $\mathrm{SP}_{2}$. As $F$ decreases continually, the trajectory gradually converges to the equilibrium point $E_{-}$and enters the quiescent state $\mathrm{QS}$ from the spiking state $\mathrm{SP}_{2}$. When the variable $F$ continues to decrease and reaches the minimum -1 , the trajectory begins to move to the right under the action of external excitation.

Similarly, due to the symmetry of the phase space, the oscillation mechanism of the trajectory moving to the right is the same as above until returning to the initial point $\mathrm{A}$ to complete a period of bursting oscillation.

In the whole process of bursting oscillation period, the system trajectory has experienced the conversion between two quiescent states and four spiking states, respectively, and the conversion between quiescent states and spiking states is caused by subcritical Hopf bifurcation of equilibrium point and fold bifurcation of limit cycle, so we can define it as symmetric subHopf/fold cycle bursting.
4.2.2. Symmetric subHopf/subHopf Bursting. Next, we set $u_{1}=0.9$ and $f=1.0$ to study the bursting oscillation and its mechanism. The phase portrait and the corresponding time history of variable $x$ are demonstrated in Figures 8(a) and 8 (b). Here, the motion of the trajectory can be divided into four parts, including two quiescent states and two spiking states.

To further reveal the bifurcation mechanism of the bursting oscillation, Figure 8(d) shows the overlap portrait of equilibrium curve and transformed phase portrait on the $(F, x)$ plane. As shown in Figure 8(d), assuming that the trajectory starts from point $\mathrm{A}$, it is attracted by the stable equilibrium point $E_{+}$. So, the trajectory moves to the left strictly along the stable equilibrium curve $E_{+}$with the decrease of $F$. At this time, the system remains in the quiescent state QS. When the trajectory moves to the subcritical Hopf bifurcation point $\operatorname{subHB}_{1}(F=0.0786)$, the equilibrium point $E_{+}$loses its stability. But, due to the delay, it still moves on for a period of time along the unstable equilibrium point $E_{+}$. Then, the trajectory jumps to the stable equilibrium point $E_{-}$due to the attraction of $E_{-}$. And the trajectory oscillates around $E_{-}$, entering the spiking state SP from the quiescent state QS. As $F$ decreases continually, the trajectory 


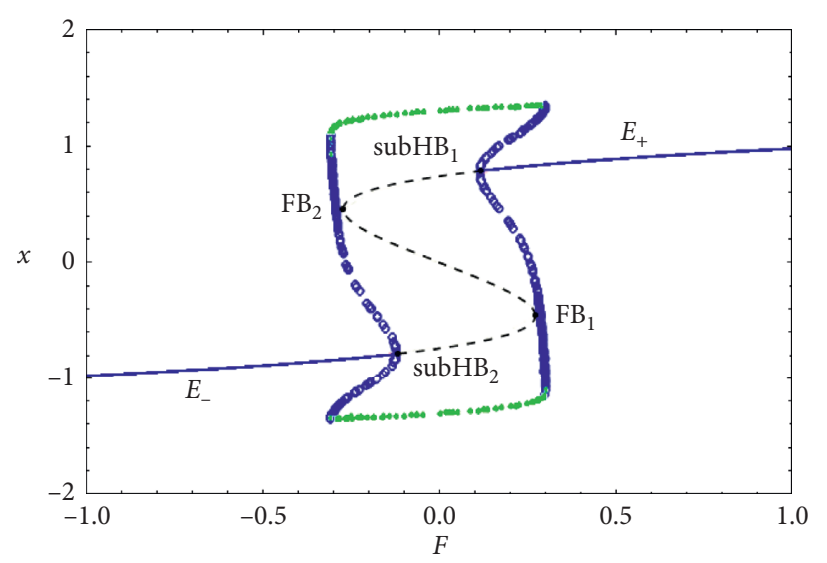

(a)

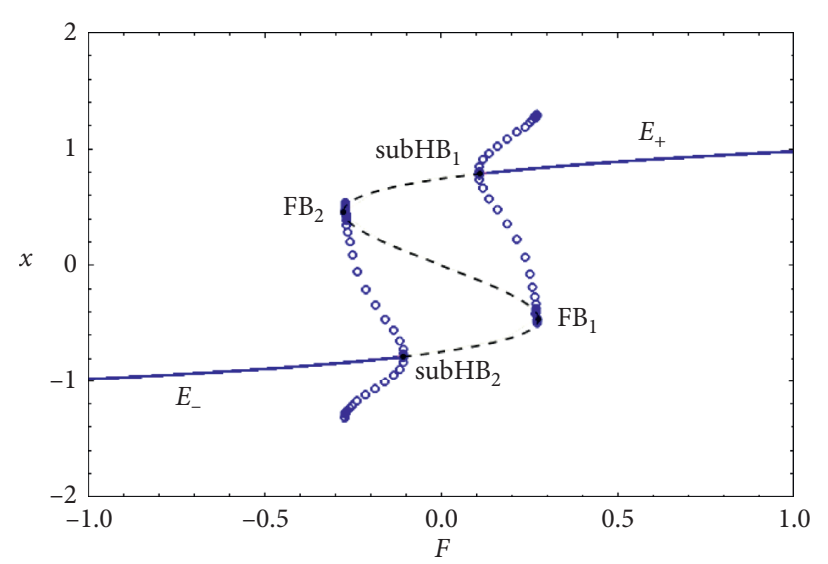

(b)

Figure 9: Equilibrium curve. (a) $u_{1}=0.85$. (b) $u_{1}=0.86$.

gradually converges to the equilibrium point $E_{-}$and enters the quiescent state QS from the spiking state SP. When the variable $F$ continues to decrease and reaches its minimum -1 , the trajectory begins to move to the right under the action of external excitation.

Similarly, due to the symmetry of the phase space, the oscillation mechanism of the trajectory moving to the right is the same as above. Until returning to the initial point $\mathrm{A}$, a period of bursting oscillation is completed.

In the whole process of bursting oscillation period, the system trajectory has experienced the conversion between two quiescent states and two spiking states, respectively, and the conversion between quiescent states and spiking states is caused by subcritical Hopf bifurcation. So, the dynamical behavior can be called symmetric subHopf/subHopf bursting.

4.2.3. CY Limit Cycles under Different $u_{1}$. For case II, different kinds of bursting oscillations can be obtained by taking different values of $u_{1}$. Figure 3 shows the equilibrium curve portrait when $u_{1}=-0.4,0.7,0.9$, and 4.0 , respectively. Therefore, we can classify the bursting oscillations in this case.

When $u_{1}<0.85$, there are stable $\mathrm{CY}$ limit cycles and unstable subcritical Hopf bifurcation limit cycles. At this point, the system will produce symmetric subHopf/fold cycle bursting oscillation.

When $u_{1} \in(0.86,3.76)$, there is no stable CY limit cycle, and there are unstable subcritical Hopf bifurcation limit cycles. At this point, the system will produce symmetric subHopf/subHopf bursting oscillation.

When $u_{1}>3.76$ (the points $\mathrm{BT}_{1,2}$ ), there is no stable $\mathrm{CY}$ limit cycle and no subcritical Hopf bifurcation point. At this point, the system will produce symmetric fold/fold bursting oscillation.

Figure 9 shows the equilibrium curve portrait when $u_{1}=$ 0.85 and 0.86 , respectively. This is the critical value of whether there are stable CY limit cycles or not. When the lower half branch of subHB $B_{1}$ touches the equilibrium curve (or the upper half branch of subHB ${ }_{2}$ touches the equilibrium curve), the stable CY limit cycles disappear and only the unstable subcritical Hopf bifurcation limit cycles exist.

\section{Conclusion}

In this paper, a Duffing-Van der Pol system with a slowvarying periodic excitation, coexistence of quintic term, and cubic term is introduced. We obtain the possible fold bifurcation set and Hopf bifurcation set by calculating system (3). Considering parameters $u_{2}, a$, and $b$, we obtain two typical parameter sets by analysis, which makes stability of equilibrium points different. Though the values of parameter sets $\left\{u_{2}, a, b\right\}$ are different, two-parameter bifurcation sets $\left(F, u_{1}\right)$ are the same. Under the two cases, adjusting the bifurcation parameters $u_{1}$ and $F$, the system will obtain different bifurcation points and produces different bursting oscillations. By analysis, we find that different $u_{1}$ and $F$ may generate the same bursting oscillations.

In order to reveal the relationship between state variables and $F$, the concept of transformed phase portrait is introduced here. Combining the transformed phase portrait with equilibrium curves portrait, we can observe bursting oscillations obviously and further reveal bifurcation mechanisms. Through bifurcation analysis, it is found that fold bifurcation and Hopf bifurcation can lead to the transition of dynamic characteristics between the spiking state and the quiescent state. Through numerical simulation, we get four kinds of bursting oscillations, namely, symmetric fold/fold bursting, symmetric fold/supHopf bursting, symmetric subHopf/fold cycle bursting, and symmetric subHopf/subHopf bursting. It is found that these four kinds of bursting oscillations are symmetric.

\section{Data Availability}

All data, models, and codes generated or used during the study are included within the article. 


\section{Conflicts of Interest}

The authors declare that there are no conflicts of interest regarding the publication of this paper.

\section{Acknowledgments}

The authors gratefully acknowledge the support of the National Natural Science Foundation of China (NNSFC) through Grant no. 11572288 and the Natural Science Foundation of Zhejiang through Grant no. LY20A020003.

\section{References}

[1] C. Kuehn, Multiple Time Scale Dynamics, Springer International Publishing, Berlin, Germany, 2015.

[2] M. Desroches, J. Guckenheimer, B. Krauskopf, C. Kuehn, H. M. Osinga, and M. Wechselberger, "Mixed-mode oscillations with multiple time scales," SIAM Review, vol. 54, no. 2 , pp. 211-288, 2012.

[3] W. Teka, J. Tabak, and R. Bertram, "The relationship between two fast/slow analysis techniques for bursting oscillations," Chaos, vol. 22, no. 4, p. 43117, 2012.

[4] M. Desroches, B. Krauskopf, and H. M. Osinga, "The geometry of slow manifolds near a folded node," SIAM Journal on Applied Dynamical Systems, vol. 7, no. 4, pp. 1131-1162, 2008.

[5] J. Guckenheimer, "Singular Hopf bifurcation in systems with two slow variables," SIAM Journal on Applied Dynamical Systems, vol. 7, no. 4, pp. 1355-1377, 2008.

[6] J. Guckenheimer and P. Meerkamp, "Unfoldings of singular Hopf bifurcation," SIAM Journal on Applied Dynamical Systems, vol. 11, no. 4, pp. 1325-1359, 2012.

[7] Y. Zhang, X. J. Han, and Q. S. Bi, "Series-mode pitchforkhysteresis bursting oscillations and their dynamical mechanisms," Chinese Journal of Theoretical and Applied Mechanics, vol. 51, no. 1, pp. 228-236, 2019.

[8] Y. Xia, Q. S. Bi, C. Luo, and X. F. Zhang, "Behaviors of modified Chua's oscillator two time scales under two excitations with frequency ratio at 1:2," Chinese Journal of Theoretical and Applied Mechanics, vol. 50, no. 2, pp. 362-372, 2018.

[9] F. Wu, H. Gu, and Y. Li, "Inhibitory electromagnetic induction current induces enhancement instead of reduction of neural bursting activities," Communications in Nonlinear Science and Numerical Simulation, vol. 79, Article ID 104924, 2019.

[10] Y. Guan, V. Gupta, and L. K. B. Li, "Intermittency route to self-excited chaotic thermoacoustic oscillations," Journal of Fluid Mechanics, vol. 894, p. R3, 2020.

[11] A. Shilnikov, "Complete dynamical analysis of a neuron model," Nonlinear Dynamics, vol. 68, no. 3, pp. 305-328, 2012.

[12] P. Channell, G. Cymbalyuk, and A. Shilnikov, "Origin of bursting through homoclinic spike adding in a neuron model," Physical Review Letters, vol. 98, no. 13, Article ID 134101, 2007.

[13] B. Bao, Q. Yang, L. Zhu et al., "Chaotic bursting dynamics and coexisting multistable firing patterns in $3 \mathrm{D}$ autonomous morris-lecar model and microcontroller-based validations," International Journal of Bifurcation and Chaos, vol. 29, no. 10, Article ID 1950134, 2019.

[14] Y. Li, H. Gu, and X. Ding, "Bifurcations of enhanced neuronal bursting activities induced by the negative current mediated by inhibitory autapse," Nonlinear Dynamics, vol. 97, no. 4, pp. 2091-2105, 2019.

[15] Z. Lü, L. Chen, and L. Duan, "Bifurcation analysis of mixed bursting in the pre-Bötzinger complex," Applied Mathematical Modelling, vol. 67, pp. 234-251, 2019.

[16] X. Guo, S. Wang, L. Sun, and D. Cao, "Dynamic responses of a piezoelectric cantilever plate under high-low excitations," Acta Mechanica Sinica, vol. 36, no. 1, pp. 234-244, 2020.

[17] X. Y. Guo, P. Jiang, D. X. Cao, and C. M. Wang, "Nonlinear vibrations of graphene piezoelectric microsheet under coupled excitations," International Journal of Non-linear Mechanics, vol. 124, Article ID 103498, 2020.

[18] J. Rinzel, "Bursting oscillations in an excitable membrane model," Ordinary and Partial Differential Equations, vol. 1151, no. 1, pp. 304-316, 1985.

[19] M. Perc and M. Marhl, "Different types of bursting calcium oscillations in non-excitable cells," Chaos, Solitons \& Fractals, vol. 18, no. 4, pp. 759-773, 2003.

[20] Q. Bi and Z. Zhang, "Bursting phenomena as well as the bifurcation mechanism in controlled Lorenz oscillator with two time scales," Physics Letters A, vol. 375, no. 8, pp. 1183-1190, 2011.

[21] X. Han, B. Jiang, and Q. Bi, "Symmetric bursting of focusfocus type in the controlled Lorenz system with two time scales," Physics Letters A, vol. 373, no. 40, pp. 3643-3649, 2009.

[22] R. Qu, Y. Wang, G. Wu, Z. Zhang, and Q. Bi, "Bursting oscillations and the mechanism with sliding bifurcations in a Filippov dynamical system," International Journal of Bifurcation and Chaos, vol. 28, no. 12, Article ID 1850146, 2018.

[23] B. C. Bao, P. Y. Wu, H. Bao, H. G. Wu, X. Zhang, and M. Chen, "Symmetric periodic bursting behavior and bifurcation mechanism in a third-order memristive diode bridgebased oscillator," Chaos, Solitons \& Fractals, vol. 109, pp. 146-153, 2018.

[24] L. Huang, G. Wu, Z. Zhang, and Q. Bi, "Fast-slow dynamics and bifurcation mechanism in a novel chaotic system," International Journal of Bifurcation and Chaos, vol. 29, no. 10, Article ID 1930028, 2019.

[25] C. Zhou, Z. Li, F. Xie, M. Ma, and Y. Zhang, "Bursting oscillations in Sprott B system with multi-frequency slow excitations: two novel "Hopf/Hopf"-hysteresis-induced bursting and complex AMB rhythms," Nonlinear Dynamics, vol. 97, no. 4, pp. 2799-2811, 2019.

[26] Z. Wen, Z. Li, and X. Li, "Bursting oscillations and bifurcation mechanism in memristor-based Shimizu-Morioka system with two time scales," Chaos, Solitons \& Fractals, vol. 128, pp. 58-70, 2019.

[27] Z. Y. Chen, X. F. Zhang, and Q. S. Bi, "Bursting phenomena as well as the bifurcation mechanism in periodically excited hartley model," Chinese Journal of Theoretical and Applied Mechanics, vol. 42, no. 4, pp. 765-773, 2010.

[28] J. K. Zheng, X. F. Zhang, and Q. S. Bi, "Bursting oscillations as well as the delayed pitchfork bifurcation behaviors in a class of chaotic system," Chinese Journal of Theoretical and Applied Mechanics, vol. 51, no. 2, pp. 540-549, 2019.

[29] J. H. Tang, X. H. Li, and Y. J Shen, "Bursting oscillation and its mechanism of van der Pol-Rayleigh system under periodic excitation," Journal of Vibration Engineering, vol. 32, no. 6, pp. 1067-1076, 2019.

[30] X. F. Zhang, X. K. Chen, and Q. S. Bi, "Relaxation bursting of a fast-slow coupled oscillator as well as the mechanism of nonsmooth bifurcation," Chinese Journal of Theoretical and Applied Mechanics, vol. 44, no. 3, pp. 576-583, 2012. 
[31] X. F. Zhang, L. Wu, and Q. S. Bi, "Bursting coexistence phenomenon of a four-dimensional non-autonomous system under periodic excitation," Journal of Dynamics and Control, vol. 14, no. 5, pp. 422-428, 2016.

[32] Z. Y. Chen, Y. G. Chen, and Q. S. Bi, "Bursting oscillations as well as the bifurcation mechanism induced by fast subsystem with multiple balances," Chinese Journal of Theoretical and Applied Mechanics, vol. 47, no. 4, pp. 699-706, 2015.

[33] X. Zhang, L. Wu, and Q. Bi, "Bursting phenomena as well as the bifurcation mechanism in a coupled BVP oscillator with periodic excitation," Chinese Physics B, vol. 25, no. 7, Article ID 070501, 2016.

[34] A. Chtouki, F. Lakrad, and M. Belhaq, "Quasi-periodic bursters and chaotic dynamics in a shallow arch subject to a fast-slow parametric excitation," Nonlinear Dynamics, vol. 99, no. 1, pp. 283-298, 2020.

[35] N. Fenichel, "Geometric singular perturbation theory for ordinary differential equations," Journal of Differential Equations, vol. 31, no. 1, pp. 53-98, 1979.

[36] E. M. Izhikevich, "Neural excitability, spiking and bursting," International Journal of Bifurcation and Chaos, vol. 10, no. 6, pp. 1171-1266, 2000.

[37] M. L. Saggio, A. Spiegler, C. Bernard, and V. K. Jirsa, "Fast-Slow bursters in the unfolding of a high codimension singularity and the ultra-slow transitions of classes," Journal of Mathematical Neuroscience, vol. 7, no. 1, pp. 1-47, 2017.

[38] X. Zhang, J. Zheng, G. Wu, and Q. Bi, "Mixed mode oscillations as well as the bifurcation mechanism in a Duffing's oscillator with two external periodic excitations," Science China Technological Sciences, vol. 62, no. 10, pp. 1816-1824, 2019.

[39] Y. Lin, W. B. Liu, H. Bao, and Q. Shen, "Bifurcation mechanism of periodic bursting in a simple three-element-based memristive circuit with fast-slow effect," Chaos, Solitons \& Fractals, vol. 131, Article ID 109524, 2020.

[40] Z. Chen and F. Chen, "Complex aperiodic mixed mode oscillations induced by crisis and transient chaos in a nonlinear system with slow parametric excitation," Nonlinear Dynamics, vol. 100, no. 1, pp. 659-677, 2020.

[41] M. Zhang and Q. Bi, "On occurrence of bursting oscillations in a dynamical system with a double Hopf bifurcation and slow-varying parametric excitations," International Journal of Non-linear Mechanics, vol. 128, Article ID 103629, 2021. 